\title{
Laser-Induced Forward Transfer-Assisted Flip-Chip Bonding of Optoelectronic Components
}

\author{
K.S. Kaur ${ }^{1}$, J. Missinne ${ }^{1}$, B. Vandecasteele ${ }^{1}$, G. Van Steenberge ${ }^{1}$ \\ 1. Centre for Microsystems Technology, IMEC/Ghent University, Technologiepark 914A, B-9052 Gent, Belgium \\ S.M.Perinchery ${ }^{2}$, E.C.P.Smits ${ }^{2}$, R.Mandamparabil ${ }^{2}$ \\ 2. TNO/Holst centre, High Tech Campus 31, AE Eindhoven, Netherlands
}

Laser-Induced Forward Transfer (LIFT) is an additive and versatile direct-write technique for a single-step pattern- definition and material transfer [1]. In LIFT, a laser pulse is focused through a transparent substrate (the carrier) onto the rear side of a thin film of the material to be printed (the donor) that transfers a donor pixel to another substrate (the receiver) placed in close proximity. In the past, LIFT has been used for printing a wide range of materials for various applications [2]. It offers several advantages such as speed, simplicity and flexibility due to which LIFT is considered to be an excellent tool for rapid prototyping especially for optoelectronic applications. In this paper, we report the first results of LIFT-assisted printing of micro-bumps of non-flux based silver nanoparticle (AgNP) and flux-based solder pastes for flip-chip bonding [3] of optoeletronic components.

The donor samples were prepared by doctor blading the respective pastes onto the quartz wafers $(2.5 \mathrm{~cm} \mathrm{x}$ $2.5 \mathrm{~cm})$. An excimer laser $(248 \mathrm{~nm}, 10 \mathrm{~ns})$ was used as the source for performing LIFT experiments. For the case of AgNP, glass substrates with pre-patterned gold bond-pads were used as receivers. Debris-free AgNP micropellets $(80 \mu \mathrm{m} \times 60 \mu \mathrm{m})$ were then LIFTed precisely on top of these pads as shown in the inset of fig.1 (a). A separation of $100 \mu \mathrm{m}$ was maintained between the donor-receiver substrates using metallic spacers. A commercially available vertical-cavity surface-emitting lasers (VCSEL) array chip (5 Gbps; $850 \mathrm{~nm} ; 1 \mathrm{x}$ 4, ) was then bonded to the receiver substrates with LIFTed micro-bumps by flip-chip bonding technique. Finally, the bonded chips were electrically characterized and fig 1 (a) depicts the typical LVI curves recorded for the VCSELs. The measured optical power matched well with the characteristics of the unbonded VCSEL chips indicating low resistance of the micro-bumops and illustrating the successful functioning of the devices. Currently, work is being carried out to bond photodiode array chips using similar approach.
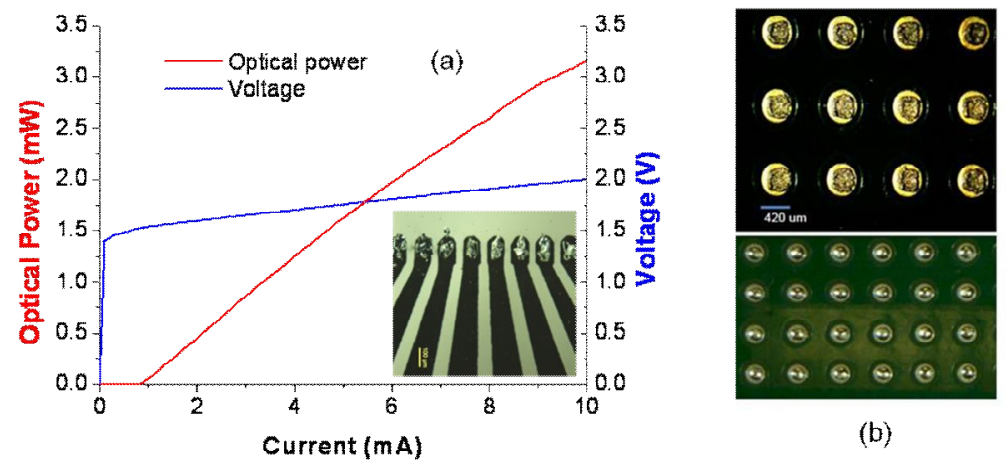

(b)

Fig. 1 (a) LVI curve recorded for one of the LIFT- assisted flip-chip bonded VCSELs. The inset shows the AgNP pixels LIFTed onto gold-bond pads. (b) Image of solder paste LIFTed on bump pads (top) and tested for reflow (bottom).

For solder paste ( $\mathrm{SnAgCu}$, type 5), polyethylene terephthalate (PET) substrates with solder bump pads (350 $\mu \mathrm{m}$ diameter) were used as receiver. Solder pixels $(300 \mu \mathrm{m} \times 300 \mu \mathrm{m})$ were then LIFTed on top of the pads keeping a separation of $35 \mu \mathrm{m}$ between the donor and receiver using Mylar spacers. The LIFTed bumps were then reflowed and the resulting pattern is shown in fig. 1 (b; bottom). It is evident from fig. 1(b) that the rosin flux was well transferred, and did not evaporate during the transfer. The reflow tests also validated the intact transfer of solder paste. Work is in progress to realize chip-bonding of light emitting diodes (LEDs) using these solder bumps.

To conclude, the successful demonstration of chip-bumping and bonding with AgNP and solder pastes using LIFT shows the great potential of the technique for flip-chip interconnect technology especially for applications requiring fine pitch and high accuracy.

\section{References}

[1] J. Bohandy, B. Kim, and F. Adrian,"Metal deposition from a supported metal film using an excimer laser," J. Appl. Phys. 60, 1538(1986). [2] C.B.Arnold, P.Serra and A.Pique, "Laser direct write techniques for printing of complex materials", MRS Bulletin, 32, 23(2007).

[3] T. Tekin, " Review of packaging of optoelectronic, photonic, and MEMS components, " J. of Select. Quantum Electron., 17, 704 (2011). 